\title{
Trauma induced adult purpura fulminans: A case report
}

\author{
Deepa Durga Roy \\ Senior Resident, Safdarjung Hospital \& V.M.M. College, New Delhi, India \\ *Corresponding Author: \\ Email: deepadurgaroy@gmail.com
}

\begin{abstract}
Introduction: Purpura fulminans is characterised by sudden and rapid advancement of hematologic and cutaneous manifestations in the form of skin necrosis and disseminated intravascular coagulation which progresses to multi organ failure resulting in death if left unidentified. Even as neonatal purpura is popularly documented, there is paucity of literature regarding adult purpura fulminans which may occur secondary to sepsis, be an autoimmune response or be of idiopathic origin. Autopsy findings are usually negative. The following case is of a young healthy army man who died suddenly of purpura fuminans consequent to a single lacerated injury sustained to lower limb.
\end{abstract}

Keywords: Purpura, Purpura fulminans, Adult, Injury, Trauma.

\section{Introduction}

Henoch in 1887 introduced the term purpura fuminans. The condition refers to rapidly progressing, life threatening, widespread, dermal microvascular thrombosis associated with disseminated intravascular coagulation (DIC) and perivascular hemorrhage characterised by non-blanch able hemorrhagic skin lesions. Purpura fuminans in neonates or children may occur in severe acute sepsis and is an important feature of meningococcemia. It may be an autoimmune phenomenon post varicella infection or may be the first phenotypic feature of heritable defects of the PC anticoagulant pathway. Purpura fulminans rarely occurs in adults. ${ }^{1}$ However, the following case is an unusual form of purpura fulminans in an adult following trauma.

\section{Case}

A 21 year old male army personel sustained injury when his motorcycle stand gave way and the bike fell on him, as a result of which he sustained a single lacerated wound in front of the left leg. The wound was surgically sutured and he was discharged from the army hospital. The following day he developed afebrile extensive painless discoloration of the whole body. The patient was a nonsmoker and non-alcoholic. He had no other significant medical history including hypertension, heart or lung disease and no prior surgical, transfusion history or hematologic disorders. There was no recent travel history. The onset of the purpura was over a period of 78 hours. On further deterioration of his condition when he started gasping, he was brought to the hospital where he was declared brought dead. On examination the deceased was an atheletic built male with a single surgically stitched wound of length $9 \mathrm{~cm}$ present $4 \mathrm{~cm}$ below knee joint in midline. The stitches were in situ. On opening the stitches the lacerated wound was muscle deep. Margins of the wound were clean. The whole body (more over the lower limbs) showed widespread non blanching reddish purple purpuric rash, which was showing bluish black hemmorhagic necrosis at places with diffuse margins, which on cut section revealed necrosis up to subcutaneous tissue. On internal examination florid petechaie were present in heart, liver and both lungs. Both the kidneys, spleen and brain were grossly normal. Skin histopathology showed thrombotic vasculopathy with extravasation of red blood cells in the dermis and subcutaneous region. This was suggestive of purpura fulminans. Cause of death was given as idiopathic adult purpura fulminans secondary to blunt injury sustained to lower limb.

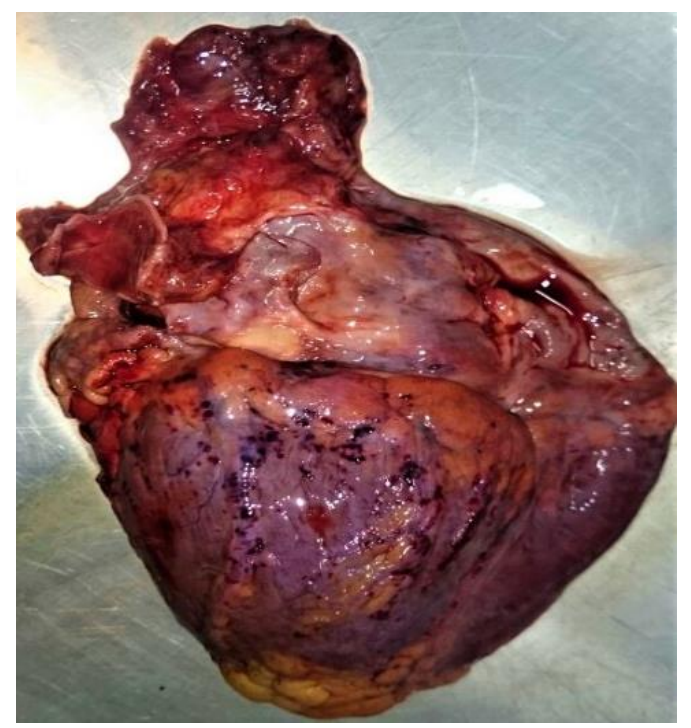

Fig. 1 


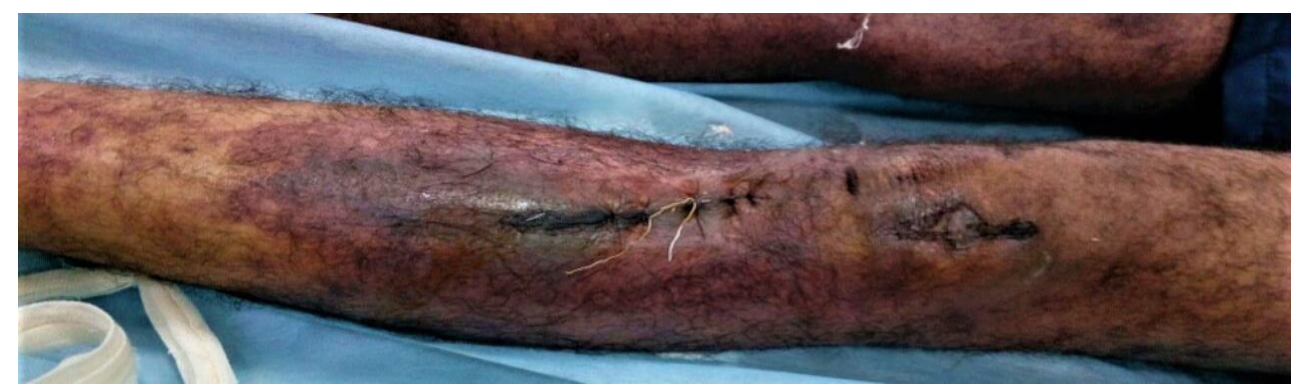

Fig. 2

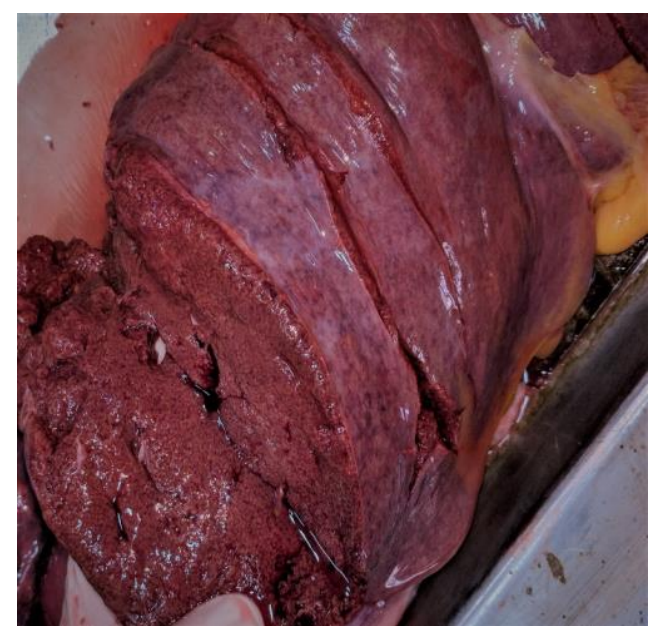

Fig. 3

\section{Discussion}

Purpura as a result of trauma has been known in the form of ecchymoses, bruising due to injury, in severe vomiting, coughing (Valsalva purpura), subconjunctival hemorrhage or eyelid or peri-orbital petechiae (Tardeiu spots) or bruising, suction to the skin (love bite), Bateman's purpura which is ecchymoses of the forearms caused by minor or even imperceptible trauma to skin fragility, as seen in elderly and/or severely sun-damaged individuals, vascular malignancy like angiosarcoma. Bruising around the mastoid bone and below the ear due to intracranial fractures referred to as Battle's sign. ${ }^{2,3}$

However, the exact mechanism leading to Purpura fulminans remains unknown. One proposed mechanism is the Shwartzman and Arthrus reaction. Purpura fulminans may be inherited homozygous protein $\mathrm{C}$ or,

Protein S deficiency; and presents itself in neonatal period, or have an idiopathic origins post varicella or scarlet fever infection or may present in illness causing sepsis with endotoxin-producing gram-negative bacteria. $^{3-6}$ The clinical manifestations are presence of non blanchable echymotic rash of skin, necrotic gangrene of the extremities with coagulation abnormalities suggestive of DIC. Histopathological findings of skin include dermal vascular thrombosis and secondary hemorrhagic necrosis. ${ }^{7}$ In the present case, the etiology of purpura fulminans seems to be secondary to trauma sustained to lower limb.

\section{Conclusion}

Purpura fulminans in adults is a rare phenomena with limited published literature, hence its clinical diagnosis is mostly unrecognised. This case demonstrates a rare presentation of purpura fulminans and hopes to make the readers aware of this entity.

\section{References}

1. Shenoy R, Nanjappa S, Eaton K, Granada CP, Messina JL, Greene JN. Purpura Fulminans: A Case Report and Review of All Causes. Infect Dis Clin Pract. 2017;25(2): 00-00

2. Sham RL, Francis CW. Evaluation of mild bleeding disorders and easy bruising. Blood Rev 1994;8:98-104.

3. Triplett DA. Coagulation and bleeding disorders: review and update. Clin Chem. 2000;46(8 Pt 2):1260-9

4. Wheeler JS, Anderson BJ, de Chalain TM. Surgical interventions in children with meningococcal purpura fulminans-a review of 117 procedures in 21 children. $J$ Pediatr Surg. 2003;38:597-603.

5. Alvarez EF, Olarte KE, Ramesh MS, et al. Purpura fulminans secondary to Streptococcus pneumonia meningitis. Case Rep Infect Dis. 2012;2012:508503.

6. Harikrishna J, Mohan A. Infectious purpura fulminans. The Indian Journal of Medical Research. 2015;141(1):130-131.

7. Adcock DM, Brozna J, Marlar RA. Proposed classification and pathologic mechanisms of purpura fulminans and skin necrosis. Semin Thromb Hemost. 1990;16:333-340. 